\title{
Preoperative Ultrasound of the MN-UA May Help to Identify Patients at Risk for latrogenic Lesion: In Response
}

I thank Dr. Kerasnoudis for the favorable comments on our study. The significance of this issue may be increased in the management of carpal tunnel syndrome (CTS). Although the sample size of the study was small, the authors demonstrated the clinical significance of the distance between the median nerve and ulnar neurovascular bundle (MN-UA) and the critical role of the ultrasonography in selecting the appropriate therapy for patients with CTS. It was interesting that there was a significant increase of the cross-sectional area (CSA) of the ulnar nerve in Guyon's canal in the group with endoscopic carpal tunnel release (E-CTR) and ulnar neuropathy at the wrist was developed in 2 of 5 patients performing E-CTS. In both patients, very short MN-UA ( $3 \mathrm{~mm}$ ) showed the importance of the preoperative ultrasonographic evaluation before E-CTR.

A considerable variation of MN-UA among individuals was established in the previous studies (1-3). However, the cause of this variation was unclear. Although our study demonstrated that median nerve swelling and flattening around the inlet of carpal tunnel might be related with the variation of $\mathrm{MN}-\mathrm{UA}$ (4), analysis be- tween MN-UA and other ultrasonographic parameters of median nerve was not performed. I did correlation analysis between MN-UA and CSA, short diameter (SD), long diameter (LD), and flattening ratio (FR) of median nerve (Table 1), which showed moderate negative correlations between MN-UA and CAS, and MN-UA and LD ( $P$ - value, $<0.001)$, and moderate positive correlation between MN_UA and FR $(P$-value, $<0.001)$.

In conclusion, it is important to recognize the change of relative distance between median nerve and ulnar neurovascular bundle probably due to median nerve swelling and flattening in patients with CTS. In CTS patients with short MN-UA, endoscopic release of carpal tunnel should be performed with caution or other therapies should be recommended.

Dr. Dong Hwee Kim, MD, PhD

Dept. of Physical Medicine andRehabilitation

Korea University College of Medicine

Ansan Hospital

516 Gojan-dong, Danwon-gu

Ansan-si, Gyeonggi Province

E-mail: rmkdh@korea.ac.kr

Table 1. Pearson's Correlation Coefficients between Ultrasonographic Parameters of the Median Nerve and the Distance between the Median Nerve and Ulnar Artery

\begin{tabular}{|c|c|c|c|c|c|c|c|c||}
\hline & \multicolumn{2}{|c|}{ CSA } & \multicolumn{2}{c|}{ SD } & \multicolumn{2}{c|}{ LD } & \multicolumn{2}{c|}{ FR } \\
\cline { 2 - 10 } & r & P-value & r & P-value & r & P-value & r & P-value \\
\hline MN-UA1 & -0.551 & 0.000 & -0.096 & 0.465 & -0.596 & 0.000 & 0.462 & 0.000 \\
\hline MN-UA2 & -0.647 & 0.000 & -0.256 & 0.049 & -0.646 & 0.000 & 0.429 & 0.001 \\
\hline MN-UA3 & -0.638 & 0.000 & -0.328 & 0.011 & -0.645 & 0.000 & 0.456 & 0.000 \\
\hline MN-UA4 & -0.565 & 0.000 & -0.259 & 0.045 & -0.668 & 0.000 & 0.427 & 0.001 \\
\hline
\end{tabular}

MN-UA1, the distance between the medial end of the median nerve and lateral end of the ulnar artery (MN-UA) at the level of $1 \mathrm{~cm}$ proximal to the distal wrist crease (DWC); MN-UA2, MN-UA at the level of $0.5 \mathrm{~cm}$ proximal to the DWC; MN-UA3, MN-UA at the level of DWC; MN-UA4, MN-UA at the level of $1 \mathrm{~cm}$ distal to the DWC; CSA, cross-sectional area of median nerve; SD, short diameter of median nerve; $\mathrm{LD}$, long diameter of median nerve; FR, flattening ratio of median nerve

\section{References}

1. Nakamichi K, Tachibana S. Distance between the median nerve and ulnar neurovascular bundle: clinical significance with ultrasonographically assisted carpal tunnel release. J Hand Surg Am. 1998; 23:870-874.
2. Frick A, Baumeister RG, Kopp R. Choice of procedure in therapy of distal median nerve compression syndrome. Handchir Mikrochir Plast Chir 1996; 28:147-150.

3. Cobb TK, Carmichael SW, Cooney WP. The ulnar neurovascular bundle at the wrist. A technical note on endoscopic carpal tunnel release. J Hand Surg $\mathrm{Br}$ 1994; 19:24-26.

4. Kim DH, Jang JE, Park BK. Anatomical basis of ulnar approach in carpal tunnel injection. Pain Physician 2013; 16:E191-198. 